\title{
La tentazione del moderno. Gesualdo Bufalino e il romanzo europeo del primo Novecento
}

The Temptation of Modernity. Gesualdo Bufalino and the European Novel of the Early 20th Century

La tentation de la modernité. Gesualdo Bufalino et le roman européen du début du $\mathrm{Xx}^{e}$ siècle

\section{Claudia Carmina}

\section{OpenEdition}

Journals

Edizione digitale

URL: http://journals.openedition.org/cei/6717

DOI: 10.4000/cei.6717

ISSN: 2260-779X

Editore

UGA Éditions/Université Grenoble Alpes

\section{Edizione cartacea}

ISBN: 978-2-37747-173-7

ISSN: 1770-9571

Notizia bibliografica digitale

Claudia Carmina, « La tentazione del moderno. Gesualdo Bufalino e il romanzo europeo del primo Novecento », Cahiers d'études italiennes [Online], 30 | 2020, Messo online il 01 mars 2020, consultato il 10 décembre 2020. URL : http://journals.openedition.org/cei/6717 ; DOI : https://doi.org/10.4000/cei. 6717

Questo documento è stato generato automaticamente il 10 décembre 2020.

(c) ELLUG 


\section{La tentazione del moderno. Gesualdo Bufalino e il romanzo europeo del primo Novecento}

The Temptation of Modernity. Gesualdo Bufalino and the European Novel of the Early 20th Century

La tentation de la modernité. Gesualdo Bufalino et le roman européen du début du $\mathrm{XX}^{e}$ siècle

\section{Claudia Carmina}

La vocazione al narrare puro, così attiva nel corso dell'Ottocento, ha dato i risultati trionfali e bellissimi che tutti abbiamo nella memoria. $\mathrm{Ma}$, a partire dalla verminosa e stupefacente Ricerca del tempo perduto, una specie di discesa nell'imbuto nella propria coscienza, in cui la narrazione viene sbriciolata negli attimi felici della reminiscenza, e a partire dall'Ulisse questa vocazione ha subito delle incrinature violente, delle quali oggi non è possibile non tener conto. Sicché la sfiducia che nasce inevitabilmente nello scrittore di fronte alle sue creature porta con sé, in modo altrettanto inevitabile, una crisi del romanzo

stesso ${ }^{1}$.

Per Bufalino la storia del romanzo moderno è segnata da una frattura. Il primo Novecento coincide con il momento di una trasformazione e di una caduta. Si sfaldano le certezze che caratterizzavano la mentalità ottocentesca, dove il mondo «era ordinato gerarchicamente con un posto proprio per ogni cosa» ${ }^{2}$. Come si legge nell'introduzione al Dizionario dei personaggi di romanzo del 1982, intitolata Passione del personaggio, tra la fine dell'Ottocento e i primi anni del nuovo secolo la letteratura registra ed elabora narrativamente i cambiamenti radicali intervenuti nell'immaginario: sono gli anni in 
cui Freud mette in crisi l'idea unitaria che l'uomo ha di sé, «Bergson frantuma in miriadi di barlumi e di schegge il diamante del conoscere, già Einstein su qualche lavagna di Berna fa stridere sotto il gesso i numeri che lo spaventano» ${ }^{3}$.

Ogni opera successiva si confronta con quel «rumore di cose che si rompono» di cui parla Virginia Woolf, con il collasso delle convenzioni narrative ottocentesche, che è il prodotto della rottura epistemologica di fine secolo e di una crisi dell'esperienza:

Le relazioni umane sono cambiate - tra servi e padroni, mariti e mogli, genitori e figli. Equando le relazioni umane cambiano, cambiano allo stesso tempo la religione, il comportamento, la politica e la letteratura. [...] Così cominciò lo sconquasso e il fracasso. Ecco perché tutto intorno a noi, in poesia, nei romanzi, nelle biografie, e perfino negli articoli di giornale, e nei saggi, sentiamo il rumore di cose che si rompono, cadono, si fracassano, e vanno distrutte. [...] Segni del genere si manifestano dappertutto. La grammatica è violata, la sintassi disintegrata ${ }^{4}$.

Muove da qui, dalla percezione di questo «sconquasso», anche la ricerca letteraria di Bufalino, in cui la «vocazione al narrare puro» porta costantemente con sé impurità e «incrinature». La fede nella letteratura convive con la consapevolezza della sua impostura. La sua scrittura replica dunque in sé la doppia tensione che attraversa la storia del romanzo «in bilico fra innocenza e malizia, certezza e ipotesi, natura e cultura ${ }^{5}$ : da una parte rimpiange nostalgicamente il mito irraggiungibile di quell'affabulazione 'assoluta' e potenzialmente infinita che si rinnova sine die nelle Mille $e$ una notte $e^{6}$ nei racconti orali dei cantastorie, nelle grandi cattedrali narrative dell'Ottocento, e in particolare nel «continente Tolstoj ch'è la natura stessa» ${ }^{7}$; dall'altra è condannata a prendere atto di uno scacco, ripercorrendo a volta a volta l'itinerario che inevitabilmente conduce allo smarrimento di ogni significato. La ricerca di senso persiste ma è destinata a restare incompiuta.

Per Bufalino la frequentazione del grande romanzo europeo assume il valore di un atto fondativo. Bufalino s'impegna nella scrittura inventiva a seguito dell'incontro folgorante con le opere di Proust, di Joyce, di Kafka, di Mann (di cui legge la Montagna incantata nella ristampa del 1945 della prima traduzione italiana) ${ }^{8}$. Diceria dell'untore, concepito subito dopo la guerra, al tempo della "glaciazione neorealista»" affonda le radici nell'esperienza parallela, insieme lacerante e rivelatrice, della malattia e della lettura appassionata del romanzo modernista, maturata ai tempi di Scandiano e poi durante la permanenza nel sanatorio di Palermo. Nell'inverno del 1944 il primario del sanatorio emiliano in cui è ricoverato gli permette di accedere alla biblioteca dell'ospedale: «[...] un ignoto marchese ha sfollato la sua biblioteca, stupenda, in un vasto abbaino del sanatorio e io vi attingo di furto, scandalizzate ma complici le suore» ${ }^{10}$, si legge in una lettera a Romanò del dicembre del 1944. Qui il giovane malato s'imbatte in un tomo della Ricerca del tempo perduto: All'ombra delle fanciulle in fiore. È un ritrovamento casuale che produce il miracolo di un «abbandono amoroso» e segna un destino:

Come se Proust fosse un infallibile, inalienabile ectoplasma di me; un nemico, magari, ma tanto incarnato da non potermene più districare; un verme solitario nelle mie viscere, un immortale ragno nella mia mente [...]. La sua lunga frase, in verità, che altro è se non il travaglio d'un gomitolo infinitesimo e sterminato, l'addipanarsi e sdipanarsi senza fine degli anelli del lombrico Tempo sotto la volta di una botola nera, che strapiomba nel silenzio? Donde una pietà si genera, vogliosa di lacrime; ma, soprattutto, una vertigine: quella che si prova di fronte a qualunque crepaccio profondo ${ }^{11}$. 
5 Scrivendo in questi mesi a Romanò, Bufalino passa in rassegna le sue scoperte di lettore: non solo Proust, ma anche Joyce ${ }^{12}$ e Kafka («Ho letto di Kafka finalmente: La Metamorfosi») ${ }^{13}$. Dalla corrispondenza emergono intuizioni e curiosità interpretative: così Bufalino progetta di scrivere un saggio, che non verrà mai alla luce, sul «"Sogno di Polifilo", una delizia linguistica del'400 che anticipa l'Ulysses nel tentativo di una prosa mescolata e polifonica» ${ }^{14}$; chiede in prestito dei testi che gli permettano di conoscere i «personaggi reali, che corrispondono ai nomi fittizi di Proust ${ }^{15}$; ipotizza che il personaggio proustiano del pittore Elstir sia in realtà una controfigura letteraria di Whistler, e che il nome ne costituisca «quasi un anagramma» 16 .

6 Un passo ancora e, dopo la guarigione, il lettore appassionato diventa a sua volta scrittore di romanzi. A lungo resta però uno scrittore 'clandestino', che ambisce ad «essere l'unico lettore di sé» ${ }^{17}$. Come la malattia, la scrittura inventiva per Bufalino è infatti uno «stigma», uno "squilibrio», uno "scandalo», da nascondere e da custodire nella dimensione del privato, e al contempo è uno «stemma» ${ }^{18}$, una segreta insegna di elezione, un privilegio solitario, un incremento simbolico.

7 Riaffiorando da questo sommerso tirocinio letterario, dopo l'uscita di Diceria dell'untore il narratore ormai sessantenne prende a pubblicare con regolarità, quasi nell'urgenza di scalfire il muro di silenzio dietro cui si era a lungo trincerato. Pubblica poesie, saggi, traduzioni, autocommenti, aforismi, un atto unico per il teatro, raccolte di racconti, e soprattutto romanzi. S'impegna in un esercizio di collaudo delle più diverse tipologie del genere: appaiono un «romanzo semiologico» ${ }^{19}$ (Argo il Cieco), un romanzo storico (Le menzogne della notte), la 'pseudo-autobiografia' Calende greche, un giallo «conforme ai canoni» (Qui pro quo), una «fiaba cavalleresca» (Il Guerrin Meschino), «un grottesco di chiacchiera e azione» (Tommaso e il fotografo cieco) ${ }^{20}$. Sono opere che nascono tra gli anni Ottanta e gli anni Novanta, quando il Postmoderno è la dominante culturale.

Bufalino costeggia il Postmodernismo letterario: ne frequenta i temi privilegiati (il labirinto, la biblioteca, il complotto, la storia e il passato come sfondi artificiali, lo scambio tra realtà e finzione, tra parole e cose); spinge in direzione della metaletteratura, della riflessione sul linguaggio, del citazionismo, dell'intertestualità. Ma l'adesione alle forme e all'immaginario postmoderno è solo parziale: libro dopo libro Bufalino continua a rilanciare una scommessa di senso. La ricerca di significato non è abolita, né il nichilismo diventa 'morbido', come invece accade di solito nella letteratura postmoderna. Permane un'ansia di significato, sebbene polverizzata, espressa sempre sotto forma di mancanza, di nostalgia per un assoluto inattingibile. La realtà è opaca, relativa, inconoscibile; eppure il pathos del significato resiste e, come ha scritto Domenica Perrone in altro contesto, si fa «tensione a trascendere il contingente in una verità universale ${ }^{21}$. Poco importa se questa verità è mobile, relativa e sempre rinegoziabile: una zigzagante scommessa sul senso del vivere e dello scrivere è continuamente rilanciata.

9 Il Postmoderno è la buccia; la polpa è la grande lezione del Modernismo di primo Novecento con cui inevitabilmente lo scrittore fa i conti ${ }^{22}$. Come nelle prove maggiori del Modernismo europeo ${ }^{23}$, anche nei libri di Bufalino il privilegio assegnato all'io convive con una trama che, per quanto svalutata, conserva una sua tenuta, dove le esperienze interiori dei personaggi contano più dei fatti oggettivi. Il narratore non è più una voce autorevole né un alfiere della verità; viceversa il suo statuto è all'insegna della bugia e della precarietà. Ad imporsi sulla pagina, ancora una volta con la 
complicità del modello modernista, è il narratore inattendibile o il «narratore che non sa $»^{24}$, che ha una versione solo parziale dei fatti o che è interno alla storia raccontata: un io dalla decisa fisionomia autobiografica che avoca a sé la totalità della funzione narrativa, ma spesso confonde menzogna e realtà, fino a smagliare la trama del racconto mettendo a nudo le frodi della letteratura. È quanto si verifica sia in Argo il cieco sia nel Guerrin meschino: in entrambi i romanzi il narratore governa i movimenti di un 'eroe-burattino' ed interviene direttamente nel testo attraverso controcanti e commenti che spezzano l'illusione del racconto, addensando sulla storia narrata l'ombra della menzogna. In ogni caso il paradigma della tradizione non è mai completamente sovvertito, bensì messo alla prova, corroso dall'interno e poi ristrutturato. Il recupero e il rinnovamento delle forme narrative passa in Bufalino attraverso una meditata scelta di stile, in cui la prosa «usurpa i privilegi della poesia» ${ }^{25}$. Così Diceria si struttura secondo una partitura sonora tendenzialmente monotonale, che procede in una musica fatta di ritmi, di pause, di accenti, e avanza per continui slittamenti metaforici, quasi con l'obiettivo di gareggiare con la «complessità ordinata» ${ }^{26}$ dello stile di Proust.

10 Il citazionismo, il rifacimento manieristico, la riscrittura producono un senso di saturazione, un sovraccarico che esprime la malinconia di fronte all'eterno ripetersi e al dissolversi del tempo. Questa tendenza intertestuale, comune all'orizzonte postmoderno, veicola però un'indagine esistenziale. L'assillo metaletterario rispecchia e argina un assillo esistenziale.

11 Una rete di riprese, allusioni e microcitazioni, tratte dai romanzi di Mann, Kafka, Proust, Joyce è disseminata tra le pagine di Diceria e delle opere successive. La citazione è per Bufalino un 'gioco serio', un tentativo di riassemblare materiali testuali precedenti in una nuova architettura di senso e in un montaggio unico. L'obiettivo è disarmare il demone della dispersione, trovare un «senso segreto» ${ }^{27}$ nella frammentarietà del reale.

12 «Un romanzo, signori, è uno specchio trasportato lungo una strada. A volte riflette l'azzurro del cielo, a volte il fango delle pozzanghere ${ }^{28}$, scriveva com'è noto Stendhal. Quello specchio si è frantumato. Per Bufalino la letteratura è uno specchio rotto in cui riconoscere il proprio sé spezzato e nel quale la vita si riflette in schegge. Parlare di altri libri è parlare di sé e del mondo: è sfidare Medusa senza guardarla direttamente, ma studiandola e aggredendola nello specchio. Questa fedeltà integrale alla letteratura ha il valore di una sfida conoscitiva ${ }^{29}$ : opporre resistenza all'assurdo, continuando a tenere ostinatamente lo sguardo fisso sull'io e sul reale, è un'esperienza assoluta e radicale in cui lo scrittore mette in gioco il proprio destino di uomo ${ }^{30}$.

13 La modalità del recupero della lezione del romanzo europeo del primo Novecento non è univoca, ma si realizza attraverso strategie diverse e complementari. Nella sua forma più esplicita e più letterale dà luogo alle pratiche della citazione e più spesso dell'allusione, la cui piena intelligenza presuppone un rapporto con un testo altro al quale si rinvia necessariamente. La piena comprensibilità dell'allusione richiede un pubblico raffinato di «febbrili lettori $i^{31}$ che, come il Robinson dell'Ingegnere di Babele, conoscano «tutti i libri», e fraziona così i livelli di ricezione «in classi non omogenee e fra di loro rivali: chi capisce, chi non capisce, chi si illude di aver capito» ${ }^{32}$. L'allusione interferisce con le ragioni della parodia; il «parlare cifrato» di Bufalino, in cui «il dissimulare è virtù e virtuosismo insieme $»^{33}$, contiene spesso una malizia autoironica. Così ad esempio, come ha notato Maria Corti ${ }^{34}$, in Argo il cieco il titolo del capitolo vi bis 
(Ritratto dell'artista come giovane zufolo) ${ }^{35}$ è un intenzionale e divertito rimando al Dedalus di Joyce; tra $\mathrm{i}$ personaggi della nobiltà modicana compare pomposamente una «duchessa Guermantes» ${ }^{36}$, ed abbondano i riferimenti alle «giovinette in fiore» («[...] se è l'addio delle giovinette in fiore, le quali, quando mi guardano, è come se guardassero un mobile da scansare») $)^{37}$.

Tuttavia, celate nelle cavità delle allusioni parodiche, affiorano genealogie più ampie $\mathrm{e}$ decisive. In Argo il cieco gli ammiccamenti divertiti a Proust segnalano una traccia intertestuale profonda: il romanzo mette in scena la lotta fra la memoria e l'oblio, riflette sulla capacità della scrittura di forzare il muro del tempo ottenendo «il miracolo del Bis, il bellissimo Riessere» ${ }^{38}$. È la stessa sfida radicale affrontata, e vinta, da Proust. La grande epifania su cui si chiude la Recherche è rappresentata dall'acquisita consapevolezza della necessità di scrivere la Recherche stessa, perché soltanto l'atto di scrittura può dare significato alla vita passata, al tempo perduto:

Si fece in me una nuova luce. E capii che erano, tutti quei materiali dell'opera, la mia vita passata; capii che erano venuti a me nei piaceri frivoli, nell'ozio, nella tenerezza, nel dolore, che li avevo immagazzinati senza capire la loro destinazione [...]. Come il seme, avrei potuto morire quando la pianta si fosse sviluppata, e mi rendevo conto d'esser vissuto per lei senza saperlo ${ }^{39}$.

Anche Bufalino affida alla scrittura il compito di penetrare il «buco grigio del Tempo» ${ }^{40}$, ma la «lucida e cosciente discesa alla Madri» - per usare l'espressione di Contini - che «avrebbe forse condotto il pellegrino alla suprema rivelazione intellettuale, ormai esente da malizia, che connota [...] Proust $»^{41}$, naufraga miseramente in Argo il cieco, mentre la spaccatura tra il moi révolu, intento a vivere le stereotipate avventure della favolosa estate del '51, ed il je actuel che prende la parola nei capitoli caudati dal bis, si allarga sempre di più, diventa divaricazione ed estraneità ${ }^{42}$. Il finale del romanzo ribalta la conclusione della Recherche e sancisce la disfatta della letteratura: «Le parole, dici... Non sono bastate, non bastano...» ${ }^{43}$.

La memoria dunque, ma anche la malattia, la 'tana', la colpa, il giudizio, la metamorfosi: è a tali nodi tematici, centrali nell'opera di Bufalino, che i meccanismi d'influenza del Modernismo europeo si legano strettamente e intrinsecamente. Le significazioni che questi temi hanno assunto ad inizio secolo in narratori come Proust, Kafka e Mann sono riproposte, indagate, sottoposte a verifica, talora confutate. Disegnano però il campo di forze intorno a cui gravita la ricerca letteraria ed esistenziale dello scrittore. Si intrecciano l'uno all'altro come parti di un tutto, crescono per accumulo e per successive stratificazioni, sono incessantemente sviscerati e riformulati, senza perdere affatto la relazione di continuità con la matrice primonovecentesca. Viceversa si impongono sulla pagina accompagnandosi sempre a una puntuale identità storicoletteraria, presentando un legame organico con precise strategie formali.

Di frequente il riferimento letterario viene introdotto attraverso l'inserto di episodi dalla forte valenza allegorica ed epifanica che sembrano compendiare per intero, enigmaticamente, il senso della narrazione. In Bufalino, così come in Kafka, l'allegoria è vuota e il suo significato resta opaco: ma proprio nella distanza che si apre tra l'«impenetrabile assurdità dell'esistenza» ${ }^{44} \mathrm{e}$ il tentativo ostinato di comprenderne il significato si gioca la sfida che Bufalino lancia all'«odiabile, amabile vita» ${ }^{45}$. Mi limito a pochi esempi tratti da Diceria dell'untore.

18 La vicenda del romanzo prende le mosse da una condanna e da un'esclusione, il cui significato resta insondabile. Marziano Guglielminetti ha notato che l'avvio del primo 
capitolo, pur entro i limiti di una più parca gestione delle varietà tonali e dei registri verbali, riecheggia la pronuncia dell'incipit della Recherche $e^{46}$ :

O quando tutte le notti - per pigrizia, per avarizia - ritornavo a sognare lo stesso sogno: una strada color cenere, piatta, che scorre con andamento di fiume fra due muri più alti della statura di un uomo; poi si rompe, strapiomba sul vuoto. Qui sporgendomi da una balconata di tufo, non trapela rumore o barlume ma mi sorprende un ribrezzo di pozzo, e con esso l'estasi che solo un irrisorio pedaggio rimanga a separarmi... Da che? ${ }^{47}$

Bufalino da un canto calibra il proprio metronomo interiore sulle movenze e sul ritmo del dettato di Proust, dall'altro innesta su questa cadenza avvolgente un richiamo perturbante. L'inizio vertiginoso di Diceria dell'untore infatti prosegue con l'immagine onirica di un redde rationem, di un processo intentato contro il protagonista-sognatore da un tribunale composto «da uomini vestiti d'impermeabili bianchi», "purgatorialmente seduti a ridosso l'uno dell'altro»". Un processo il cui «verdetto è certo» anche se è «oscura la colpa»" ${ }^{49}$. La dinamica descritta nel sogno è la stessa di un altro, più noto processo: quello apocalittico allestito nei confronti di Josef $\mathrm{K}$. in una Praga da «Vecchio Testamento» ${ }^{50}$. Nel Dizionario dei personaggi di romanzo, tratteggiando i ritratti dei personaggi kafkiani, Bufalino ne mette a fuoco il medesimo intreccio tra malattia e colpa che risulta cruciale in Diceria dell'untore:

\section{$1912 \quad$ Gregorio Samsa}

Svegliarsi una mattina cambiati in un sozzo insetto, e a chi non è successo una volta? Gregorio Samsa, dunque, se ne stupisce meno che non ne soffra. Gli toccherà assuefarsi a convivere con questa infezione nascosta, esiliarsi nella vergogna delle sue inette e striscianti zampine, finché la morte non lo consoli: metafora di uno stato di alienità e solitudine senza speranza, da cui si leva, fra macchie d'escrementi e bave, pietosamente un'implorazione ${ }^{51}$.
1922
L'agrimensore $\mathrm{K}$.

Una malattia, quando giunge, è un'espiazione cercata, quantomeno meritata. Allo stesso modo chi può credere seriamente che Josef, Gregorio, K. subiscano processo, metamorfosi e interdizione, senza averli oscuramente voluti? Nessuno è innocente dunque? [...] Forse vivere è contemporaneamente una pena e una colpa, e forse il colpevole di vivere è punito appunto col vivere, per quanti fortilizi di tana si muri attorno fingendo d'esser morto. E K., l'agrimensore, misurerà presto gli sconfinati confini di un'eterna colonia penale ${ }^{52}$.

La perspicuità del nodo che lega l'io narrante di Diceria a Josef K., all'agrimensore K. e a Gregor Samsa emerge con evidenza anche dalle note preparatorie dell'intervento che Bufalino tiene il 27 ottobre 1990 al Teatro Vittorio Emanuele di Messina, intitolato Da stigma a stemma. Il malato come eroe letterario. Qui lo scrittore passa in rassegna il campionario di 'malattie letterarie' della storia della cultura occidentale, nella convinzione che la malattia, nella doppia valenza di «colpa» e di «privilegio», possa essere elevata a figura stessa della letteratura. Stendendo in vista dell'intervento un elenco di 'malati letterari', Bufalino annota sotto la rubrica «Il Malato metaforico»: «Kafka / Io Diceria», inserendo i due riferimenti uno di seguito all'altro, in un'unica catena semantica ${ }^{53}$. La malattia e la colpa che gravano sull'io narrante di Diceria richiamano dunque un'altra malattia, un'altra colpa. Eppure il riferimento a Kafka non basta a costruire un ordine di verità per la scena minacciosa che apre Diceria: fornisce sì una chiave interpretativa all'allegoria, ma questa rimane doppiamente, enigmaticamente vuota.

21 Altri due elementi tematici contribuiscono a caratterizzare il Kafka di Bufalino: la metamorfosi e l'autoisolamento in una 'tana' che, come il sanatorio di Diceria, è «trono 
e trappola $»^{54}$. «La provincia: fodero, castello d'Atlante, prigione...? "Come potrei vivere in un'aria che non fosse quella del carcere?" (Kafka)» ${ }^{55}$ : in uno degli aforismi del Malpensante Bufalino utilizza la citazione di Kafka per dar voce alla propria predilezione letteraria e psicologica per gli spazi chiusi, già esplicitamente rivendicata anche in un'intervista del 1981:

Sarà per un vizio da sanatorio (ma prima c'erano stati la casa, la caserma, il ventre materno), certo è che la tana è il mio luogo di elezione. Del resto, nella mia opera, il tema della clausura volontaria, dell'autosequestro, è ricorrente: la Rocca in Diceria, il castello-prigione nelle Menzogne, la stanza-nascondiglio in Felicità del bambino punito (nell'Uomo invaso e poi in Calende)... ancora in Calende un capitolo s'intitola Carcere d'invenzione, piranesianamente... ${ }^{56}$

A questa lista di luoghi letterari va aggiunta la Comiso di Museo d'ombre, «un paese infetto, dunque, un paese infelice» ${ }^{57}$. Arricchiscono il catalogo i due spazi chiusi in cui si svolge la trama doppia di Argo il cieco: la stanza dell'albergo romano da cui il vecchio narratore racconta la sua storia e la Modica della giovinezza che, anch'essa, «finisce con l'essere un luogo chiuso» ${ }^{58}$; e ancora vanno ricordate le ambientazioni dei romanzi più tardi: 'Le Malcontente' in cui è ambientato Qui pro quo; Flower City, il condominio di Tommaso e il fotografo cieco. Nel cuore di questa geografia si colloca infine un luogo reale che ha la consistenza di una prigione simbolica: la Sicilia.

Capire la Sicilia significa dunque per un siciliano capire se stesso, assolversi o condannarsi. Ma significa, insieme, definire il dissidio fondamentale che ci travaglia, l'oscillazione tra claustrofobia e claustrofilia, fra odio e amor di clausura, secondo che ci tenti l'espatrio o ci lusinghi l'intimità di una tana, la seduzione di vivere la vita come un vizio solitario. L'insularità, voglio dire, non è una segregazione solo geografica, ma se ne porta dietro altre: della provincia, della famiglia, della stanza, del proprio cuore. Di qui il nostro orgoglio, la diffidenza, il pudore; e il senso di essere diversi ${ }^{59}$.

Una «tana prudente» ${ }^{60}$, un cadavere in decomposizione, un'arca scampata al diluvio, un carcere, un luogo d'incantesimi: queste sono le immagini che Bufalino usa con maggiore frequenza per rappresentare il microcosmo dalla Rocca. L'ambientazione romanzesca in un sanatorio, cioè in uno dei luoghi più frequentati dalla letteratura del primo Novecento, inserisce Diceria all'interno di una precisa mappa intertestuale, che ha al centro La montagna incantata di Mann.

Bufalino dialoga a distanza con Mann, recuperando dalla Montagna incantata la grammatica della malattia come "vizio» ma anche come «privilegio». Intorno al campo semantico della malattia si intesse una trama metaforica a maglie strette che, attraverso il ritorno di alcune parole-chiave, lega la vicenda di Diceria al romanzo manniano. In entrambe le opere il malato è più volte paragonato a un «novizio» e la malattia è descritta al modo di un' «iniziazione». Così per Naphta l'ingresso in sanatorio ricorda un rito iniziatico e l'esperienza della tubercolosi permette di accedere ad un «mistero» ignoto ai sani:

Il discepolo deve essere animato da sete di sapere. [...] La fossa, la tomba, fu sempre l'emblema principale della solennità del patto. Il discepolo, colui che desidera essere iniziato al sapere, deve dimostrare davanti ad essa il suo coraggio. Gli usi dell'Ordine vogliono che egli, per prova, sia fatto discendere nella fossa e vi rimanga per poi uscirne guidato dalla mano di un fratello sconosciuto. [...] La via dei misteri e della purificazione era circondata da pericoli, conduceva attraverso la paura della morte, il regno della putrefazione; e il discente, il neofita, era la gioventù avida dei miracoli della vita $[. . .]^{61}$. 
Non diversamente per Gesualdo Bufalino l'attesa della morte è una «iniziazione quasi amorosa» ${ }^{62}$, un «apprendistato di morte» ${ }^{63}$, una «veglia d'armi, preparazione mistica ad un rito d'investitura», e il moribondo è un «catecumeno del nulla» ${ }^{64}$.

Il Berghof è un "covo del vizio» ${ }^{65}$; anche la vita alla Rocca è un «vizio» da cui però «ci vuole poco a svezzarsi $»^{66}$. E tuttavia per i personaggi di Mann e di Bufalino la guarigione coincide con una «diserzione» e un «tradimento». Una volta guarito, l'io narrante di Diceria ha la percezione di aver compiuto un' «infrazione» ${ }^{67}$, violando un "patto» segreto:

Questo nei primi mesi, poi all'esistenza smozzicata degli altri finii con l'assuefarmi, e dal loro consorzio non volli più disertare. [...] Ma se di tanti io solo, premio o pena che sia, sono scampato e respiro ancora, è maggiore il rimorso che non il sollievo, d'aver tradito a loro insaputa il silenzioso patto di non sopravviverci ${ }^{68}$.

Il sollievo, il rimorso, il senso di una violazione e di una colpa, la necessità di giustificarsi dinanzi ai compagni di degenza: queste pulsioni contraddittorie erano già state sperimentate dal personaggio di Joachim nella Montagna incantata. Quando Joachim decide di abbandonare la dimensione scarna e rarefatta del Berghof, Naphta, Hans e Behrens giudicano la sua partenza al modo di un'incomprensibile «diserzione»:

«Ma, dica, devo credere alle mie orecchie? Lei vuol deporre le armi e andarsene? Ma sa che questo si chiama disertare! $»^{69}$.

Laggiù in pianura, avrebbe dovuto svolgere la sua vita, fra la gente che non aveva idea alcuna di come si dovesse vivere, che nulla sapeva di termometro, dell'arte di avvolgersi nelle coperte, di introdursi nel sacco a pelo, nulla delle tre passeggiate regolamentari diuturne. Era difficile enumerare tutto ciò che non si sapeva laggiù [...]. «Non la ritengo tale», aveva detto Gioachino quando Radamanto aveva parlato di diserzione. Ma per lui, il borghese, le cose stavano diversamente. Per lui la partenza era senz'altro una diserzione! ${ }^{70}$.

Nella Montagna incantata l'antitesi tra un «su» (la montagna) e un "giù» (la pianura) rappresenta, sul piano dello spazio, l'opposizione tra malattia e salute. La montagna su cui sorge il Berghof ospita il mondo chiuso e larvale dei malati; la pianura è lo spazio aperto e attivo in cui agiscono i sani. Al Berghof, in montagna, il tempo è immobile, come cristallizzato; in pianura invece scorre sempre più accelerato, fino a precipitare rovinosamente nell'imbuto della guerra.

In Diceria i valori spaziali si ribaltano. Tra interno ed esterno non c'è scarto. Il sanatorio e la città sono entrambi luoghi corrotti. Non c'è alternativa alla malattia. Fuori dal sanatorio non c'è salvezza.

Così, mentre il Berghof è un osservatorio che consente di guardare il mondo dall'alto' di una distanza critica, la Rocca è invece, alla maniera di Kafka, una tana, un' «isola, fodero ${ }^{71}$, abitata da miasmi e contagi, in cui anche l'amore si tinge di morte. L'innamoramento dell'io narrante per Marta si mescola a un gusto necrofilo. Anche l'amore è miasma e contagio. Il protagonista possiede di Marta non una fotografia, bensì una radiografia.

Perché contro ogni creanza e verità io m'ostinavo a presumere d'aver tacitamente stretto un patto con lei, e di possederne caparra nella radiografia trafugata che tenevo sotto il cuscino. Questa, mi bastava accarezzarla con un dito, la sera e ne ricavavo un aggricciarsi agrodolce di nervi, quale dà a qualcuno la setagloria di un parapioggia, se gli sfiora per caso i capelli. Al punto che quell'esile celluloide, contro cui s'era premuto con forza il suo petto, piuttosto che continuare a sembrarmi, come all'inizio, la tela filata di una tarantola scura, s'era venuta mutando, non meno che guanto o stivaletto, in una sorta di inaudito feticcio amoroso... ${ }^{72}$ 


\section{tempo all'interno di Diceria ${ }^{76}$.}


no è tramato da una rete di citazioni e di rimandi intertestuali (i più appariscent dei quali dialogano con Montale, come ha dimostrato Giuseppe Traina) ${ }^{78}$. Ancora una volta davanti al lettore si spalanca l'abisso di una parabola indecifrabile. Ancora una volta l'apologo allegorico ha come sottotesto Kafka, e qui in particolare il brevissimo racconto Dalla galleria, in cui viene messo in scena lo spettacolo crudele di una artista «cadente e tisica» che, come Marta, rischia la vita per portare a termine il suo numero:

Se una qualsiasi cavallerizza cadente e tisica venisse fatta girare senza interruzione nel maneggio sopra un cavallo traballante, davanti a un pubblico instancabile, dal direttore spietato agitante la frusta, mentre essa lancia baci e si dondola sulle anche, e se questo giuoco continuasse fra il perpetuo strepito dell'orchestra e dei ventilatori verso il grigio avvenire che si spalanca senza fine, accompagnato dall'applauso delle mani che si spegne e di nuovo rinasce, delle mani che sono veramente magli a vapore, allora forse un giovane frequentatore della galleria si affretterebbe a scendere per la lunga scalinata attraverso tutti gli ordini, si precipiterebbe nel maneggio e griderebbe: «Alt!» attraverso le fanfare dell'orchestra che sempre si adatta.

Ma siccome non è così, una bella dama, bianca e rossa, vola dentro, tra le tende che gli orgogliosi macché aprono davanti a lei ${ }^{79}$.

Quello di Kafka è un racconto doppio. La situazione iniziale, tracciata in questo incipit, presenta la cavallerizza malata, costretta ad esibirsi da un direttore sadico, fino a che un giovane spettatore interviene a interrompere lo spettacolo che rischia di uccidere la donna; la seconda parte del racconto però nega la prima: la cavallerizza, questa volta sana e gioiosa, esegue il suo numero sotto lo sguardo affettuoso del direttore, mentre il giovane spettatore, incapace di agire e inchiodato a guardare da una posizione di esclusione, alla fine dell'esibizione scoppia in un pianto inesplicabile ${ }^{80}$. Bufalino fa rivivere nell'episodio del ballo di Marta la stessa terna di personaggi (la donna artista, lo spettatore, il direttore) e comunica uno stesso duplice pathos: lo spettatoreinterprete assiste all'«aerea scrittura» della donna con una partecipazione e una concentrazione assolute, perché percepisce nella scena un senso che lo riguarda ma resta misterioso; nella sua immaginazione è lui a salvare la donna, permettendole di concludere il balletto, ma nella realtà assiste immoto allo spettacolo. Il palcoscenico è il luogo di una metamorfosi imperfetta: la ballerina si trasforma in una sorta di «angelo nunciante», ma il lettore non sa se la metamorfosi sia una condanna o una salvezza.

È la stessa ambiguità che grava sulla metamorfosi descritta nel racconto che dà il titolo alla raccolta L'uomo invaso. Qui a narrare in prima persona è il protagonista, Vincenzino La Grua, vittima e beneficiario di una metamorfosi e di un'interdizione oscuramente volute, che lo mutano in un angelo:

Da qualche tempo lo sospettavo, anche se mi mancava il coraggio di parlarne con qualcuno. Ma ora l'evidenza mi sopraffà: io Vincenzino La Grua, non sono più un uomo ma un angelo, probabilmente un serafino ${ }^{81}$.

L'incipit exabrupto mette il lettore di fronte all'evidenza di una trasformazione inesplicabile, strizzando l'occhio all'inizio bruciante, e tanto più perturbante, della Metamorfosi («Quando Gregor Samsa si risvegliò una mattina da sogni tormentati si ritrovò nel suo letto trasformato in un insetto gigantesco») ${ }^{82}$.

Vincenzino, come la ballerina di Diceria, è un doppio dello scrittore e insieme è una parodia in scala minore di Gregor Samsa. In entrambi i casi, nel racconto e nel romanzo, l'allegoria resta silenziosa. Rinvia ad un significato che è individuale e problematico, e non offre al lettore una chiave di lettura prestabilita. Nel vuoto dei 
significati universali, validi per tutti, si limita a segnalare uno strappo non più ricucibile tra l'io e la realtà. Il narratore può solo mostrare il trauma dell'insignificanza. Gli stessi personaggi vorrebbero disperatamente che la loro storia significasse qualcosa, alludesse ad una verità 'altra' e più universale. Ma questo 'altro' non si rivela; come accade in Kafka e nei grandi romanzi del Modernismo europeo, il senso è diventato inaccessibile. Allo scrittore non resta che interpretare la condizione di spettatore estraneo, affannandosi però tenacemente nell'interrogare i frammenti di un senso possibile, alla ricerca convulsa di un significato, ostinandosi a scommettere sulla forza della letteratura.

\section{NOTE}

1. Gesualdo Bufalino: autoritratto con personaggio, intervista di M. Onofri a G. Bufalino, in G. Bufalino, Opere/2 1989.1996, a cura di F. Caputo, Milano, Bompiani, 2007, pp. 1341-1342.

2. R. Kern, Il tempo e lo spazio. La percezione del mondo tra Otto e Novecento, Bologna, Il Mulino, 2007, p. 259.

3. G. Bufalino, Passione del personaggio, in Id., Opere/2 1989.1996, cit., p. 1178.

4. V. Woolf, Voltando pagina. Saggi 1904-1941, a cura di L. Rampello, Milano, Il Saggiatore, 2011, pp. 122 e 133.

5. G. Bufalino, Morire a Roncisvalle, «Sigma», $\mathrm{n}^{\circ} 33,1984$, p. 66.

6. La centralità assunta dalle Mille e una notte nell'opera dello scrittore comisano è stata messa in luce da Marina Paino, in L'ombra di Sheherazade. Suggestioni dalle «Mille e una notte» nel Novecento italiano, Cava de' Tirreni, Avagliano Editore, 2004, pp. 113-143.

7. G. Bufalino, Passione del personaggio, cit., p. 1178.

8. Una copia del volume di Mann nella traduzione italiana ristampata nel 1945 è consultabile nella biblioteca appartenuta allo scrittore e oggi conservata presso la Fondazione Bufalino di Comiso (cfr. T. Mann, La montagna incantata, traduzione dal tedesco di B. Giachetti-Sorteni, Milano, Dall'Oglio Editore, 1945; la stessa traduzione di Bice Giachetti-Sorteni era stata pubblicata per la prima volta nel 1930 dalla casa editrice Modernissima di Milano).

9. G. Bufalino, Istruzioni per l'uso, in Id., Opere 1981.1988, a cura di M. Corti e F. Caputo, Milano, Bompiani, 1992, p. 1299.

10. G. Bufalino, lettera ad A. Romanò dell'1 dicembre del 1944, in A. Romanò e G. Bufalino, Carteggio di gioventù (1943-1950), a cura di N. Zago, Valverde, Il Girasole, «Dioniso», 1994, p. 53. La lettera dell'1 dicembre è riportata anche in G. Bufalino, Opere/2 1989.1996, cit., p. 1284.

11. G. Bufalino, Cere perse, in Id., Opere 1981.1988, cit., p. 1011. Per quanto riguarda il rapporto di Bufalino con Proust, stabilito «all'insegna di un'ideale e spontanea consonanza», si rilegga quanto scrive Marina Paino in Dicerie dell'autore. Temi e forme della scrittura di Bufalino, Firenze, L.S. Olschki, 2005, pp. 74 e sgg.; e in Ead., L'ombra di Sheherazade. Suggestioni dalle «Mille e una notte» nel Novecento italiano, cit., pp. 125-133.

12. Cfr. G. Bufalino, lettere ad A. Romanò del 23 aprile, del 6 luglio, dell'1 dicembre del 1944, in A. Romanò e G. Bufalino, Carteggio di gioventù (1943-1950), cit., rispettivamente alle pp. 44, 56, 60. 13. G. Bufalino, lettera ad A. Romanò del 13 maggio 1944, in A. Romanò e G. Bufalino, Carteggio di gioventù (1943-1950), cit., p. 49. 
14. G. Bufalino, lettera ad A. Romanò dell'1 dicembre 1944, in A. Romanò e G. Bufalino, Carteggio di gioventù (1943-1950), cit., p. 56.

15. Ibid.

16. Ibid.

17. G. Bufalino, Il malpensante, in Id., Opere 1981.1988, cit., p. 1067.

18. G. Bufalino, Da stigma a stemma. Il malato come eroe letterario, intervento letto a Messina il 27 ottobre 1990, in occasione della Cerimonia Inaugurale del XCI Congresso Nazionale della Società Italiana di Medicina Interna, Edizioni Cepi, Roma, 1990; ora in Id., Opere/2 1989.1996, cit., p. 1215.

19. È Bufalino stesso a definire Argo il cieco «un romanzo di strutture e segni, un romanzo semiologico» (G. Bufalino, Cur? Cui? Quis? Quomodo? Quid?, Atti del wordshow-seminario sulle maniere e le ragioni dello scrivere, Taormina, Edizioni «Agorà», 1989, p. 60).

20. Queste sono le definizioni di genere che, nei rispettivi risvolti di copertina, Bufalino assegna a Qui pro quo, al Guerrin Meschino, a Tommaso e il fotografo cieco (cfr. Note ai testi, in G. Bufalino, Opere/ 2 1989.1996, cit., pp. 1402, 1410, 1421). Un profilo complessivo di Bufalino, che resta prezioso e imprescindibile, è stato tracciato da Nunzio Zago, in Gesualdo Bufalino. La figura e l'opera, Marina di Patti, Pungitopo, 1987.

21. D. Perrone, I sensi e le idee, Palermo, Sellerio, 1985, p. 23.

22. Per 'storicizzare' la produzione letteraria di Bufalino, si rileggano le osservazioni di Nunzio Zago che riflette sul rapporto tra la «formazione High Modern» dell'autore e le suggestioni postmoderniste delle ultime opere nel saggio I sortilegi della parola. Studi su Gesualdo Bufalino, Leonforte, Euno Edizioni, 2016, in particolare alle pp. 25-26 e 125-132.

23. Per ripercorrere il dibattito sul modernismo italiano cfr. L. Somigli e M. Moroni (a cura di), Italian Modernism: Italian Culture between Decadentism and Avant-Garde, Toronto, University of Toronto Press, 2004; M. Tortora, La narrativa modernista italiana, «Allegoria», $\mathrm{n}^{\circ}$ 63, lug.-dic. 2010, pp. 83-91, poi ripubblicato in Letteratura e politiche culturali. Note critiche sul Novecento italiano, Perugia, Morlacchi, 2012; R. Luperini e M. Tortora (a cura di), Sul modernismo italiano, Napoli, Liguori, 2012; P. Pellini, Naturalismo e modernismo. Zola, Verga e la poetica dell'insignificante, Roma, Artemide, 2016; R. Donnarumma - S. Grazzini (a cura di), La rete dei modernismi europei. Riviste letterarie e canone (1918-1940), Perugia, Morlacchi, 2016; R. Luperini, Dal modernismo a oggi. Storicizzare la contemporaneità, Roma, Carocci, 2018; L. Somigli e E. Conti (a cura di), Oltre il canone: problemi, autori e opere del modernismo italiano, Perugia, Morlacchi, 2018; M. Tortora (a cura di), Il modernismo italiano, Roma, Carocci, 2018.

24. M. Tortora, La novella, in Id. (a cura di), Il modernismo italiano, cit., p. 53.

25. G. Bufalino, Cur? Cui? Quis? Quomodo? Quid?, cit., p. 24.

26. M. Proust, I Guermantes, in Id., Alla ricerca del tempo perduto, a cura di M. Bongiovanni Bertini, Torino, Einaudi, 1978, p. 51.

27. Cfr. "Oscillo sempre fra due proposizioni estreme e contrarie: se scrivere sia solo un giocattolo, una medicina, insomma un succedaneo che aiuta a subire 1a vita; oppure il senso segreto d'essa» (G. Bufalino, Bluff di parole, in Id., Opere/2 1989.1996, cit., p. 54).

28. Stendhal, Il rosso e il nero, Roma, Newton Compton, 1994, p. 226.

29. Su questo aspetto cfr. N. Zago, I sortilegi della parola. Studi su Gesualdo Bufalino, cit., pp. 30-31.

30. Si rilegga a questo proposito quanto scrive Traina sulla «letteratura al quadrato» in Bufalino: per lo studioso l'intertestualità non è concepita dallo scrittore comisano come divertissement ma al modo di un «universo/soluzione alternativa che consenta di preservare l'umanità dell'uomo» (G. Traina, Siciliani ultimi? Tre studi su Sciascia, Bufalino e Consolo. E oltre, Modena, Mucchi, 2014, p. 26).

31. Cfr. «Febbrile lettore, s'interrompeva di quando in quando per ricopiare una frase su un notes, indi ricominciava. Un cliente senza nome, mi dissero, ma al bar lo chiamavano Robinson» (G. Bufalino, L'uomo invaso, in Id., Opere 1981.1988, cit., p. 465). 
32. G. Bufalino, Cur? Cui? Quis? Quomodo? Quid?, cit., p. 44.

33. G. Bufalino, Cere perse, in Id., Opere 1981.1988, cit., p. 895.

34. M. Corti, Introduzione a G. Bufalino, Opere 1981.1988, cit., p. Xx.

35. G. Bufalino, Argo il cieco ovvero I sogni della memoria, in Id., Opere 1981.1988, cit., p. 293. Si noti come in Argo il cieco Bufalino faccia sempre seguire il punto al titolo del singolo capitolo. Non è un caso: ititoli, in genere lunghi e denotativi, funzionano al modo di rubriche introduttive. Il romanzo ha una architettura calibrata e doppia: diciassette capitoli ospitano il racconto dell'estate felice trascorsa a Modica nel 1951 da un professore trentenne, la cui vicenda è rievocata retrospettivamente dallo stesso protagonista ormai anziano; sette capitoli (III bis, VI bis, VIII bis, XI bis, XII bis, XVII bis, XVII ter), più un'iniziale Locandina delle intenzioni, si presentano invece come controcanti in cui l'io narrante vecchio e malato, in una stanza d'albergo a Roma, racconta il suo tentativo di scrivere quello stesso libro «felice» che il lettore ha tra le mani.

36. Ivi, p. 301.

37. Ivi, p. 351. Per pedinare più approfonditamente il filo delle allusioni proustiane presenti in Argo il cieco si rimanda al saggio di An de Neve, Gesualdo Bufalino e il palinsesto proustiano, in M. Bastiaensen et al., Tempo e memoria nella lingua e nella letteratura italiana, vol. III, Narrativa del Novecento e degli anni Duemila, Bruxelles, «Civiltà italiana», $\mathrm{n}^{\circ}$ 5, 2009, pp. 121-132. Un divertito rimando a Proust ritorna anche in Qui pro quo, nell'incipit del quarto capitolo: «Andati tutti a letto presto la sera» (G. Bufalino, Qui pro quo, in Id., Opere/2 1989.1996, cit., p. 232).

38. G. Bufalino, Cere perse, in Id., Opere 1981.1988, cit., p. 823.

39. M. Proust, Il Tempo ritrovato, in Id., Alla ricerca del tempo perduto, edizione diretta da L. De Maria e annotata da A. Beretta Anguissola e D. Galateria, traduzione di G. Raboni, vol. IV, Milano, Mondadori, «I Meridiani», 1998, p. 582.

40. G. Bufalino, Cere perse, in Id., Opere 1981.1988, cit., p. 823.

41. G. Contini, Saggio introduttivo, in C. E. Gadda, La cognizione del dolore, Torino, Einaudi, 1963, pp. 10-11.

42. La presenza della discrepanza tra moi révolu e je actuel, di cui parla in altro contesto Jean Starobinski (L'occhio vivente, Torino, Einaudi, 1975), è stata già messa in evidenza da Rosa Maria Monastra nella sua analisi di Diceria dell'untore (cfr. R. M. Monastra, La «Diceria dell'untore», ovvero il perturbante esorcizzato con rito letterario, «Le forme e la storia», $\mathrm{n}^{\mathrm{i}}$ 1-2, 1981).

43. G. Bufalino, Argo il cieco ovvero I sogni della memoria, cit., p. 398.

44. G. Debenedetti, Il romanzo del Novecento. Quaderni inediti, Milano, Garzanti, 1987, p. 515.

45. G. Bufalino, Argo il cieco ovvero I sogni della memoria, cit., p. 400.

46. Cfr. M. Guglielminetti, Il codice autobiografico della Diceria dell'untore, in N. Zago (a cura di), Bufalino narratore fra cinema, musica, traduzione, Comiso, Salarchi Immagini, 2002, p. 200.

47. G. Bufalino, Diceria dell'untore, in Id., Opere 1981.1988, cit., p. 9.

48. Ivi, p. 10. Sull'incipit di Diceria cfr. G. Traina, «La felicità esiste, ne ho sentito parlare». Gesualdo Bufalino narratore, Cuneo, Nerosubianco, 2012, pp. 11 e sgg.; e A. Di Grado, «Che mastro, questo don Gesualdo!», in N. Zago (a cura di), Bufalino narratore fra cinema, musica, traduzione, cit., pp. 215 e sgg.

49. G. Bufalino, Dizionario dei personaggi di romanzo. Da Don Chisciotte all'Innominabile, Milano, Bompiani, 2000, p. 385.

50. Ibid.

51. Ivi, p. 371.

52. Ivi, p. 412

53. F. Caputo, Note ai testi, in G. Bufalino, Opere/2 1989.1996, cit., p. 1464.

54. G. Bufalino, Cur? Cui? Quis? Quomodo? Quid?, cit., p. 79.

55. G. Bufalino, Il malpensante, in Id., Opere 1981.1988, cit., p. 1082.

56. Gesualdo Bufalino: autoritratto con personaggio, cit., p. 1321.

57. G. Bufalino, Due fotografi a Comiso, cent'anni fa, in Id., Opere/2 1989.1996, cit. p. 1099. 
58. Cfr. "[Modica è] una piccola cittadina che finisce con l'essere un luogo chiuso» (Un giallo, anzi uno scherzo, intervista a G. Bufalino di G. Bonina, «La Sicilia», 16 aprile 1996).

59. G. Bufalino, La luce e il lutto, in Id., Opere 1981.1988, cit., p. 1141.

60. G. Bufalino, Cur? Cui? Quis? Quomodo? Quid?, cit., p. 79.

61. T. Mann, La montagna incantata, traduzione di B. Giachetti-Sorteni, Milano, Dall'Oglio Editore, 1945, p. 578.

62. G. Bufalino, Istruzioni per l'uso, in Id., Opere 1981.1988, cit., p. 1297.

63. G. Bufalino, Diceria dell'untore, in Id., Opere 1981.1988, cit., p. 9.

64. G. Bufalino, Cur? Cui? Quis? Quomodo? Quid?, cit., p. 122.

65. T. Mann, La montagna incantata, cit., p. 710.

66. G. Bufalino, Diceria dell'untore, in Id., Opere 1981.1988, cit., p. 139.

67. G. Bufalino, Istruzioni per l'uso, in Id., Opere 1981.1988, cit., p. 1298.

68. G. Bufalino, Diceria dell'untore, in Id., Opere 1981.1988, cit., p. 25.

69. T. Mann, La montagna incantata, cit., p. 471.

70. Ivi, pp. 474 e 475.

71. G. Bufalino, Istruzioni per l'uso, in Id., Opere 1981.1988, cit., p. 1298.

72. G. Bufalino, Diceria dell'untore, in Id., Opere 1981.1988, cit., p. 55.

73. Cfr.: «Era il ritratto interno di Claudia che, senza volto, mostrava la delicata struttura ossea del suo tronco ravvolto nelle morbide forme della carne, forme trasparenti e misteriose, e gli organi della cavità toracica... Quante volte l'aveva contemplato premendolo poi sulle labbra durante il tempo che era trascorso da allora, e mentre esso portava i suoi mutamenti!» (T. Mann, La montagna incantata, cit., p. 397).

74. Ivi, p. 667.

75. G. Bufalino, Diceria dell'untore, in Id., Opere 1981.1988, cit., rispettivamente alle pp. 111, 86, 93.

76. Cfr.: «Egualmente, il capitolo sul ballo di Marta nacque autonomamente, quando ancora non esistevano né il personaggio né la trama del romanzo. Poi ho abbandonato, ripreso, aggiunto; le modifiche insomma sono state nel corso degli anni» (Gesualdo Bufalino: autoritratto personaggio, cit., p. 1324).

77. G. Bufalino, Diceria dell'untore, cit., pp. 41-43.

78. G. Traina, Presenze linguistiche e tematiche della poesia montaliana in "Diceria dell'untore» di Gesualdo Bufalino, «Siculorum Gymnasium», n. s., a.XLII, n ${ }^{\mathrm{i}} 1-2$, gennaio-dicembre 1990, pp. 239-271.

79. F. Kafka, Dalla galleria, in Id., I racconti, traduzione di H. Furst, Milano, Longanesi, 1965, p. 155. L'edizione citata è quella posseduta da Bufalino, oggi conservata presso la Fondazione Bufalino di Comiso.

80. Per un'analisi approfondita del racconto di Kafka si rimanda al saggio di Romano Luperini, Lo spettatore, la cavallerizza e l'incontro impossibile, in Id., L'incontro e il caso. Narrazioni moderne e destino dell'uomo occidentale, Bari-Roma, Laterza, 2017, pp. 288-308.

81. G. Bufalino, L'uomo invaso, in Id., Opere 1981.1988, cit., p. 408.

82. F. Kafka, La Metamorfosi, in Id., Tutti i racconti, cit., p. 47. 


\section{RIASSUNTI}

La scrittura di Gesualdo Bufalino dialoga con i grandi modelli della narrativa primonovecentesca europea (Proust su tutti). Una rete di riprese, allusioni e microcitazioni, tratte dai grandi romanzi di Mann, Kafka, Proust, Musil, Joyce, Woolf, è disseminata tra le pagine di Diceria dell'untore, che nasce come un 'gioco serio' di ricombinazione di materiali testuali precedenti, rielaborati in una nuova architettura di senso. Come nelle prove maggiori del modernismo europeo, anche nei libri successivi di Bufalino il privilegio assegnato all'io convive con una trama che, per quanto svalutata, conserva una sua tenuta, dove le esperienze interiori dei personaggi contano più dei fatti oggettivi. Persiste un'urgenza di verità e di significato. Poco importa se questa verità è mobile, relativa e sempre rinegoziabile: una zigzagante scommessa sul senso del vivere e dello scrivere è sempre rilanciata, pagina dopo pagina. L'assillo esistenziale si traduce in un assillo metaletterario che recupera la lezione, i modi e la struttura del romanzo modernista.

The work of Gesualdo Bufalino relies on a permanent dialogue with the great modernist models of European fiction. A whole network of allusions, innuendos and micro-quotations from the great novels of Mann, Kafka, Proust, Musil, Joyce, Woolf, is scattered among the pages of Diceria dell'untore, a novel which was conceived as a "serious game" using previous textual materials and recombining them in order to create a new architecture of meaning. In Bufalino's following books as well, the central position assigned to the Ego coexists with a plot in which the inner experiences of the characters count more than the objective facts. There is an urgency of truth and meaning. The fact that this truth is fluid, relative and always renegotiable does not really matter: page after page, the author keeps betting on the meaning of living and writing. The existential predicament turns itself into a meta-literary predicament that is nurtured by the traditions and structures of the modernist novel.

L'écriture de Gesualdo Bufalino dialogue avec les grands modèles de la fiction moderniste européenne et en premier lieu avec Proust. Un réseau d'allusions et de micro-citations, renvoyant aux grands romans de Mann, Kafka, Proust, Musil, Joyce, Woolf, est disséminé parmi les pages de Diceria dell'untore, qui se présente comme un "jeu sérieux " où des matériaux textuels précédents sont recombinés et réinscrits dans une nouvelle architecture de sens. De même, dans les livres suivants de Bufalino, les expériences intérieures des personnages comptent plus que les faits objectifs, mais la trame garde une certaine consistance, à l'instar des grandes créations du modernisme européen. Vérité et sens ne perdent rien de leur urgence. Peu importe que cette vérité soit mobile, relative et renégociable : un pari zigzaguant sur le sens de la vie et de l'écriture est constamment relancé, page après page. L'obsession existentielle se traduit par une obsession métalittéraire qui récupère les leçons et les structures narratives du roman moderniste.

\section{INDICE}

Mots-clés : Proust, Mann, Kafka, Joyce, Woolf, Diceria dell'untore, modernisme, roman, citation, métamorphose, thème de la tanière, thème de la maladie, culpabilité, mémoire

Parole chiave : Gesualdo Bufalino, Proust, Mann, Kafka, Joyce, Woolf, Diceria dell'untore, modernismo, primo Novecento, romanzo, citazione, Claudia Carmina, metamorfosi, tema della tana, tema della malattia, tema della colpa, memoria, romanzo modernista

Keywords : Proust, Mann, Kafka, Joyce, Woolf, Diceria dell'untore, Modernism, novel, quotation, metamorphosis, theme of the den, theme of the disease, guilt, memory 


\section{AUTORE}

CLAUDIA CARMINA

Università di Palermo 